\title{
Spectral and waveform characteristics of fine and coarse crackles
}

Mitsuru Munakata, Hideaki Ukita, Isamu Doi, Yoshinori Ohtsuka, Yoshitaka Masaki, Yukihiko Homma, Yoshikazu Kawakami

\section{Abstract}

Two acoustically different types of lung crackles, fine and coarse, occur in different pathophysiological conditions. To differentiate these crackles from objective characteristics of frequency information, crackles were recorded from 16 patients with pulmonary fibrosis judged clinically to have "fine" crackles and from 10 with chronic bronchitis who had mainly "coarse" crackles. Time expanded waveforms (1/4 cycle duration, initial deflection width, two cycle duration, and $9 / 4$ cycle duration; duration of the first $1 / 4,2 / 4,8 / 4$, and $9 / 4$ cycles of crackle waveforms) were examined and fast Fourier transform analysis (peak and maximum frequencies) was performed. All waveform measurements for fine crackles were significantly smaller than those for coarse crackles. Peak and maximum frequencies for fine crackles were significantly higher than those for coarse crackles. Although there was some overlap in these values for individual crackles between the two groups when average values of these measurements were calculated for each patient, there was no overlap between fine and coarse crackles and the two groups could be clearly separated. Log peak frequency and $\log$ maximum frequency correlated better with $9 / 4$ cycle duration $(r=0.85,0.84)$ and two cycle duration $(r=0.87,0.86)$ than with $1 / 4$ cycle duration $(r=0.66,0.77)$ or initial deflection width $(r=0.67,0.79)$. Early and late segments of crackles have different characteristics, probably related to the origin of the sound and the resonance of the lung respectively. These results suggest that spectral and waveform characteristics may help to improve the accuracy of pulmonary auscultation and increase knowledge of how crackles are generated.

First Department of Medicine, School of Medicine, Hokkadio University, Sapporo 060, Japan

M Munakata

H Ukita

I Doi

Y Ohtsuka

Y Masaki

Y Homma

Y Kawakami

Reprint requests to:

Dr Munakata

Accepted 19 June 1991

Two acoustically different types of lung crackles are recognised clinically. ${ }^{1-4}$ The American Thoracic Society recommends the use of the terms fine and coarse for these crackles. ${ }^{2}$ Fine crackles occur in patients with interstitial lung disease and early congestive heart failure, are heard during inspiration, and are high pitched..$^{3-7}$ They were thought to be produced by peripheral airway opening during inspiration, ${ }^{3-6}$ and this has been confirmed experimentally. ${ }^{8}$ Coarse crackles occur in patients with chronic bronchitis and severe pulmonary oedema, are heard during both inspiration and expiration, and are low pitched. ${ }^{46}$ Fluid secreted in the airways is thought to contribute to the production of coarse crackles. ${ }^{4}$ Because the different types of crackles are due to different diseases, it is important to differentiate these two types. Some of the characteristics of the crackles, such as pitch, are based on subjective description, and pulmonary auscultation is unreliable and subject to personal variability. It would be valuable therefore if measurements of pitch could be used to separate the two types of crackle objectively. Time expanded waveform analysis was proposed for this purpose. ${ }^{9}$ The following measurements have been recommended by Holford ${ }^{10}$ and Matsuzaki ${ }^{11}$ (fig 1): the durations of the first half cycle (initial deflection width), of two cycles (two cycle duration), of the first $1 / 4$ cycle of crackles ( $1 / 4$ cycle duration), and of the first $9 / 4$ cycles $(9 / 4$
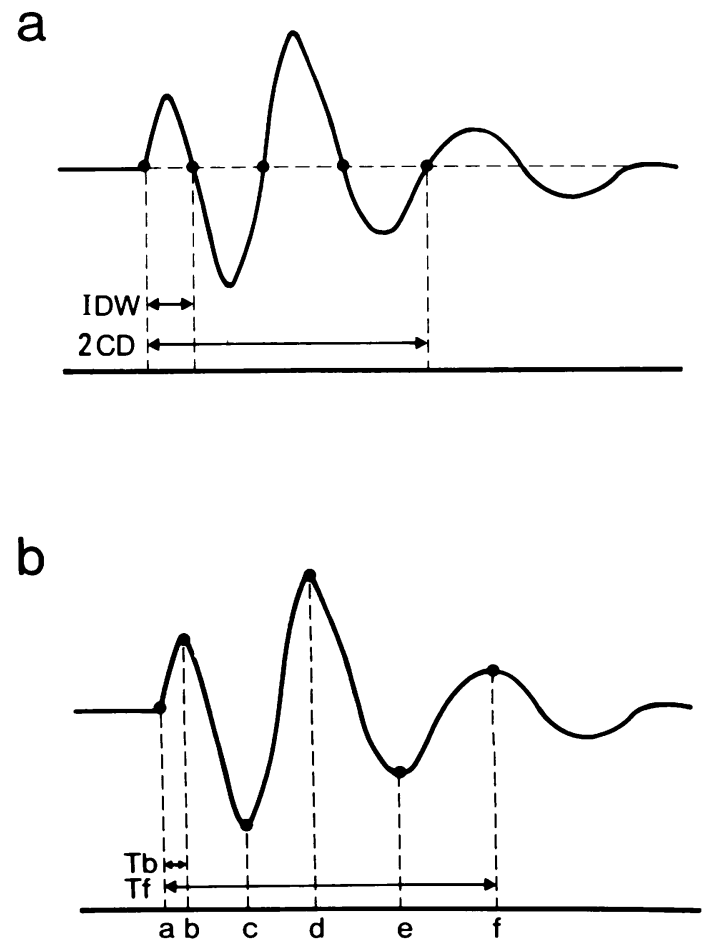

Figure 1 Measurements in time expanded waveform of the first half cycle of the crackle waveform) and two cycle duration (2CD: the duration of the first two cycles of the crackle waveform). (b) Tb (the duration of the first $1 / 4$ cycle of the crackle waveform) and Tf (the duration of the first $9 / 4$ cycles of the crackle waveform). analysis. (a) Initial deflection width (IDW: the duration 
Figure 2 Schematic representation of the measurement system. MIC-microphone; $A M P$-amplifier; $D A T$ digital audio tape recorder; $A / D$-analogdigital converter; FFTfast Fourier transform.

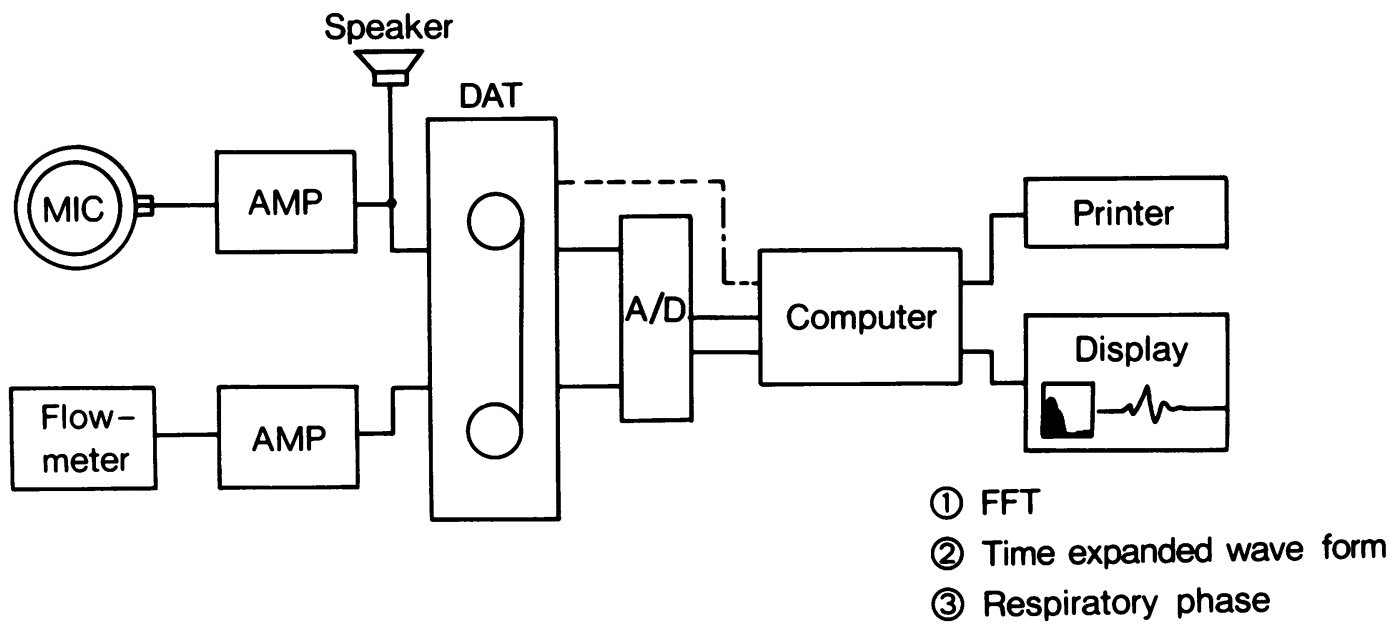

cycle duration). Fast Fourier transform analysis is another objective measurement of sound pitch, but there are technical difficulties in applying this to very short waveforms.

The purpose of the present study was to develop a system to perform time expanded waveform and fast Fourier transformation analysis for each crackle. We then applied the method to describe the spectral and waveform characteristics of crackles in patients with idiopathic pulmonary fibrosis and chronic bronchitis.

\section{Methods \\ DEFINITION OF CRACKLES}

Because in the original description of fine and coarse crackles $^{25}$ their pitch was described as high and low respectively (without objective criteria) it would have been inappropriate to define and select patients on the basis of fine and coarse crackles. We therefore studied two types of crackles, defined as follows: (1) crackles heard only during inspiration in patients with uncomplicated idiopathic pulmonary fibrosis, no sputum production, and no expiratory crackles (fine crackles); (2) crackles in patients with chronic bronchitis with expectoration of sputum (coarse crackles).

\section{SELECTION OF THE PATIENTS}

We studied 16 patients selected from 67 patients with idiopathic pulmonary fibrosis diagnosed at Hokkaido University Hospital from 1976 to 1988. All patients had the following diagnostic criteria or had histological confirmation of the diagnosis: progressive dyspnoea without spirometric evidence of airway obstruction, bilateral crackles over the lungs, and bilateral interstitial shadows on the chest radiograph. Sarcoidosis, allergic alveolitis, and other known causes were excluded by detailed investigations, including transbronchial lung biopsy. Histological confirmation of the diagnosis by open lung biopsy or necropsy was obtained in five of the 16 patients.

We also studied 10 patients with chronic bronchitis with daily sputum production. All patients had the following features: cough and sputum (at least $50 \mathrm{ml} /$ day) for at least three months for two consecutive years, airways obstruction (a low ratio of forced expiratory volume to vital capacity, $\mathrm{FEV}_{1} / \mathrm{FVC}$ ), coarse crackles, and normal transfer factor for carbon monoxide.

\section{SAMPLING OF CRACKLES} condenser microphone (Sony ECM 150) and analysed with a newly developed lung sound analyser (fig 2). The system was constructed with an eight channel digital audio tape recorder (Sony DAT PC-108M) and computer (NEC-PC9801). Sounds were introduced into the digital audio tape recorder and converted from analogue to digital signals simultaneously with flow information from a pneumotachograph. Sampling frequency was $20 \mathrm{kHz}$, and the sampling time was 6.55 seconds. Lung sounds were usually recorded throughout full inspiration at the base of the right posterior chest wall in order to minimise interference from heart sounds. Because crackles recur in consecutive respiratory phases ${ }^{57}$ sampling was limited to a single inspiratory phase for each patient. Five crackles from one inspiratory phase for each patient were sampled randomly and analysed.

\section{ANALYSES OF CRACKLES}

The time expanded waveform analysis and the fast Fourier transform analysis were performed with the Wave Master program (Canopus Electronics Company Ltd, Kobe, Japan). For time expanded waveform analysis sound signals were usually displayed at $1 \mathrm{~ms} /$ division and 100 $\mathrm{mV} /$ division on the screen (fig 3 ). The duration of the first $1 / 4$ and $9 / 4$ cycles for each crackle, ${ }^{811}$ the initial deflection width (duration of the first half cycle), and the duration of the first two cycles $^{10}$ (fig 1 ) were analysed on the screen by pointing cursors at two points on the waveform, from which the computer calculated the time between the two points.

For frequency analysis fast Fourier transform analysis was performed for each crackle waveform. Sampling frequency was $20 \mathrm{kHz}$ and the window for fast Fourier transform analysis was adjusted to $51.2 \mathrm{~ms}$ ( 1024 points). To eliminate background noises and artefacts the single waveform signal was extracted by cutting at two zero points, before and after the
Lung sounds were recorded with an electric 

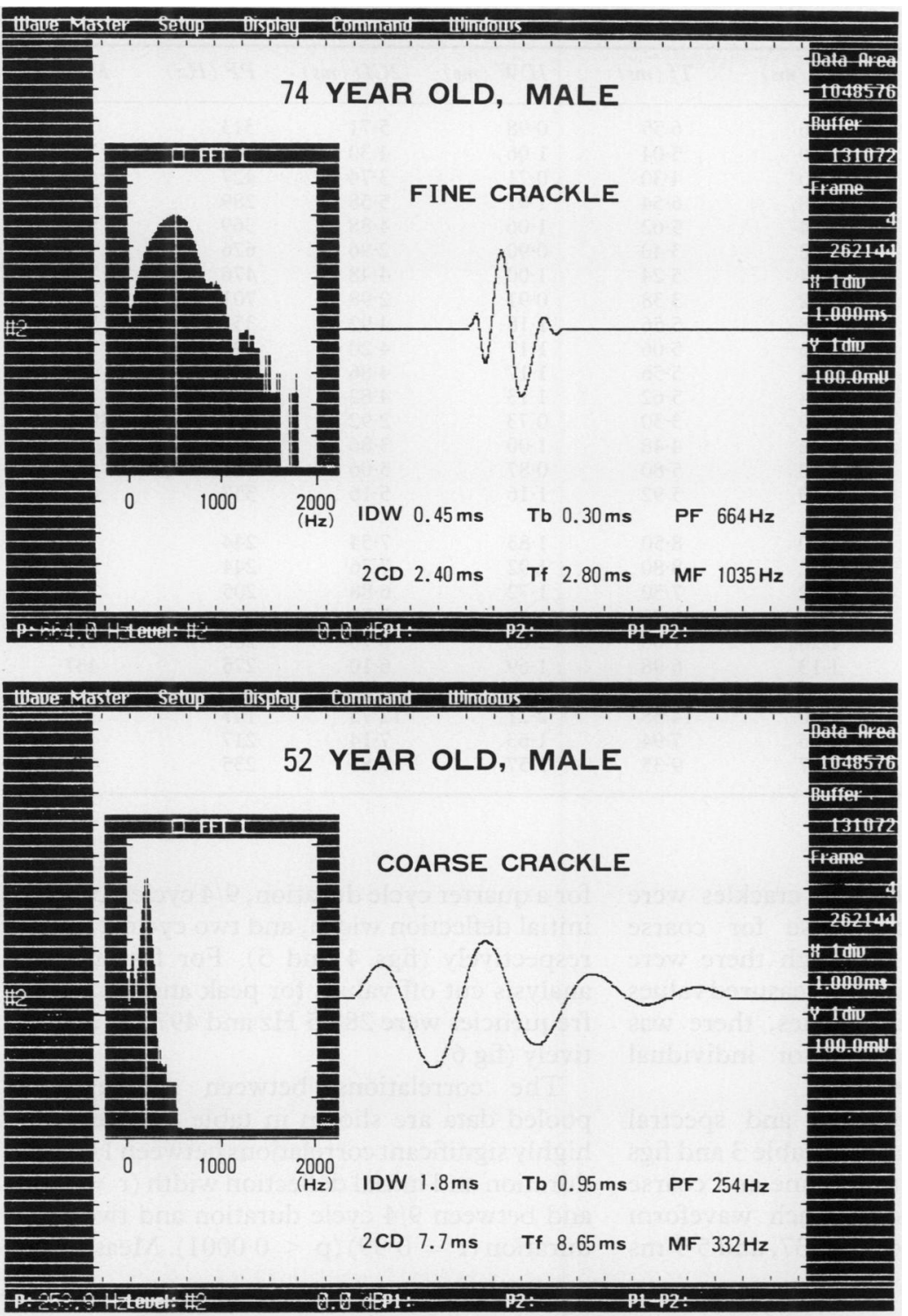

Figure 3 Example of time expanded waveform and fast Fourier transform analysis of fine and coarse crackles with the system: (a) fine crackle; (b) coarse crackle. PF-peak frequency; MF-maximum frequency. Other abbreviations as in figure 1.

waveform, and inserted into a continuous zero baseline. Then the window for fast Fourier transform analysis was applied to include the waveform in the middle of the zero baseline. A Hanning window was applied to the $51.2 \mathrm{~ms}$ analysis window and fast Fourier transformation was performed. The frequency measurements were peak frequency and maximum frequency (frequency at $-20 \mathrm{~dB}$ of the amplitude at peak frequency). They were obtained by placing cursors on the peak of the spectrum and on the point $-20 \mathrm{~dB}$ from the peak amplitude on the screen, which automatically indicated the frequency of each point of the spectrum (fig 3 ).

\section{STATISTICAL ANALYSIS}

The means and standard errors of the mean of each measurement for the two groups were calculated and compared by means of Student's $t$ test. In addition, the mean values for the five crackles from each patient were compared. Correlations between the different measurements were obtained for all crackles, log-
Table 1 Anthropometric and pulmonary functional data (mean ( $S D$ ) values)

\begin{tabular}{|c|c|c|}
\hline & $I P F$ & $C B$ \\
\hline Age (y) & $62 \cdot 3(10 \cdot 7)$ & $46.0(10.8)$ \\
\hline Height $(\mathrm{cm})$ & $159.0(6.0)$ & $162.9(6.8)$ \\
\hline Weight (kg) & $63.0(9.4)$ & $51 \cdot 6(5 \cdot 7)$ \\
\hline VC (\% pred) & $82 \cdot 1(20 \cdot 5)$ & $59.5(7.5)$ \\
\hline $\mathrm{FEV}_{1} / \mathrm{FVC}(\%)$ & $82 \cdot 8(7 \cdot 0)$ & $58.1(5.9)$ \\
\hline TLCo (\% pred) & $66 \cdot 5(36 \cdot 6)$ & $83.9(23 \cdot 6)$ \\
\hline $\mathrm{PaO}_{2}(\mathrm{~mm} \mathrm{Hg})$ & $78 \cdot 0(15 \cdot 6)$ & $65.9(8.4)$ \\
\hline $\mathrm{PaCO}_{2}(\mathrm{mmHg})$ & $39 \cdot 3(4 \cdot 0)$ & $40 \cdot 1(4 \cdot 7)$ \\
\hline
\end{tabular}

IPF-idiopathic pulmonary fibrosis; $\mathrm{CB}$-chronic bronchitis; VC-vital capacity; $\mathrm{FEV}_{1}$-forced expiratory volume in one second; FVC-forced vital capacity; TLCO-carbon monoxide transfer factor; $\mathrm{PaO}_{2}$-arterial oxygen tension; $\mathrm{PaCO}_{2}-$ arterial carbon dioxide tension.

arithmic transformation being performed for peak and maximum frequencies. Analysis of the difference between two correlation coefficients was performed as proposed by Hotelling. ${ }^{12}$ When $p$ values were less than 0.05 the differences were considered to be significant.

\section{Results}

Anthropometric and pulmonary function data for the patients are shown in table 1. Patients with chronic bronchitis were younger and lighter $(p<0.05)$. Patients with idiopathic pulmonary fibrosis had a lower transfer factor for carbon monoxide than those with chronic bronchitis. Patients with chronic bronchitis had a lower $\mathrm{FEV}_{1} / \mathrm{FVC}$ and arterial oxygen tension.

Mean values for the sound measurements are shown in table 2 . Five crackles from a single inspiratory phase were sampled from all patients with idiopathic pulmonary fibrosis and from eight of 10 patients with chronic bronchitis; in two patients only four crackles could be sampled. Thus 80 crackles were analysed from the patients with idiopathic pulmonary fibrosis and 48 from the patients with chronic bronchitis. The quarter cycle duration, $9 / 4$ cycle duration, initial deflection width, and two cycle duration for "fine" crackles were significantly shorter $(p<0.0001)$ than those for "coarse" crackles. Peak and

Table 2 Mean (SE) values for each measurement of crackles from patients with idiopathic pulmonary fibrosis ("fine") and chronic bronchitis ("coarse")

\begin{tabular}{lcc}
\hline & $\begin{array}{l}\text { "Fine" } \\
(n=80)\end{array}$ & $\begin{array}{l}\text { "Coarse" } \\
(n=48)\end{array}$ \\
\hline $\mathrm{Tb}(\mathrm{ms})$ & $0 \cdot 63(0 \cdot 02)^{\star}$ & $1 \cdot 16(0 \cdot 03)$ \\
$\mathrm{Tf}(\mathrm{ms})$ & $5.059(0.16)^{\star}$ & $8 \cdot 79(0 \cdot 38)$ \\
$\mathrm{IDW}(\mathrm{ms})$ & $1.00(0 \cdot 03)^{\star}$ & $1 \cdot 88(0 \cdot 05)$ \\
$2 \mathrm{CD}(\mathrm{ms})$ & $4 \cdot 40(0 \cdot 14)^{\star}$ & $7 \cdot 74(0 \cdot 32)$ \\
PF $(\mathrm{Hz})$ & $443(16)^{\star}$ & $233(8)$ \\
MF $(\mathrm{Hz})$ & $721(20)^{\star}$ & $394(10)$ \\
\hline
\end{tabular}

$\mathrm{Tb}-1 / 4$ cycle duration; IDW-initial deflection width; $2 \mathrm{CD}$ - two cycle duration; $\mathrm{Tf}-9 / 4$ cycle duration (the duration of the first $1 / 4,2 / 4,8 / 4$, and $9 / 4$ cycles of crackle waveforms); $\mathrm{PF}$-peak frequency; MF-maximum frequency.

*Significantly different from "coarse crackles" $(\mathrm{p}<0.0001), t$ test). 
Table 3 Mean values for waveform and spectral measurements for each patient

\begin{tabular}{llllllll}
\hline Patient No & No of crackles & $T b(m s)$ & $T f(m s)$ & $I D W(m s)$ & $2 C D(m s)$ & $P F(H z)$ & $M F(H z)$ \\
\hline IPF-1 & 5 & $0 \cdot 56$ & $6 \cdot 55$ & $0 \cdot 98$ & $5 \cdot 71$ & 313 & 732 \\
IPF-2 & 5 & $0 \cdot 60$ & $5 \cdot 04$ & $1 \cdot 06$ & $4 \cdot 30$ & 469 & 732 \\
IPF-3 & 5 & $0 \cdot 40$ & $4 \cdot 30$ & $0 \cdot 71$ & $3 \cdot 74$ & 427 & 669 \\
IPF-4 & 5 & $0 \cdot 68$ & $6 \cdot 54$ & $1 \cdot 01$ & $5 \cdot 58$ & 289 & 549 \\
IPF-5 & 5 & $0 \cdot 74$ & $5 \cdot 62$ & $1 \cdot 06$ & $4 \cdot 88$ & 369 & 590 \\
IPF-6 & 5 & $0 \cdot 62$ & $3 \cdot 40$ & $0 \cdot 90$ & $2 \cdot 96$ & 676 & 928 \\
IPF-7 & 5 & $0 \cdot 64$ & $5 \cdot 24$ & $1 \cdot 00$ & $4 \cdot 48$ & 476 & 791 \\
IPF-8 & 5 & $0 \cdot 62$ & $3 \cdot 38$ & $0 \cdot 91$ & $2 \cdot 98$ & 701 & 918 \\
IPF-9 & 5 & $0 \cdot 78$ & $5 \cdot 56$ & $1 \cdot 18$ & $4 \cdot 92$ & 351 & 527 \\
IPF-10 & 5 & $0 \cdot 66$ & $5 \cdot 06$ & $1 \cdot 17$ & $4 \cdot 20$ & 391 & 730 \\
IPF-11 & 5 & $0 \cdot 78$ & $5 \cdot 56$ & $1 \cdot 17$ & $4 \cdot 86$ & 364 & 593 \\
IPF-12 & 5 & $0 \cdot 74$ & $5 \cdot 62$ & $1 \cdot 13$ & $4 \cdot 82$ & 471 & 713 \\
IPF-13 & 5 & $0 \cdot 49$ & $3 \cdot 30$ & $0 \cdot 73$ & $2 \cdot 92$ & 620 & 1052 \\
IPF-14 & 5 & $0 \cdot 62$ & $4 \cdot 48$ & $1 \cdot 00$ & $3 \cdot 86$ & 450 & 711 \\
IPF-15 & 5 & $0 \cdot 50$ & $5 \cdot 80$ & $0 \cdot 87$ & $5 \cdot 06$ & 361 & 672 \\
IPF-16 & 5 & $0 \cdot 70$ & $5 \cdot 92$ & $1 \cdot 16$ & $5 \cdot 16$ & 357 & 632 \\
& & & & & & \\
CB-1 & 5 & $1 \cdot 10$ & $8 \cdot 50$ & $1 \cdot 83$ & $7 \cdot 54$ & 244 & 371 \\
CB-2 & 5 & $1 \cdot 12$ & $8 \cdot 80$ & $1 \cdot 92$ & $7 \cdot 76$ & 244 & 381 \\
CB-3 & 5 & $1 \cdot 02$ & $7 \cdot 50$ & $1 \cdot 72$ & $6 \cdot 88$ & 205 & 371 \\
CB-4 & 5 & $1 \cdot 18$ & $8 \cdot 78$ & 1.98 & $7 \cdot 54$ & 222 & 415 \\
CB-5 & 5 & $1 \cdot 28$ & $7 \cdot 60$ & $2 \cdot 06$ & $6 \cdot 76$ & 288 & 417 \\
CB-6 & 4 & $1 \cdot 13$ & $6 \cdot 98$ & $1 \cdot 69$ & $6 \cdot 10$ & 275 & 467 \\
CB-7 & 5 & $1 \cdot 34$ & $8 \cdot 66$ & $2 \cdot 20$ & $7 \cdot 80$ & 247 & 349 \\
CB-8 & 4 & $1 \cdot 40$ & $14 \cdot 58$ & $2 \cdot 21$ & $12 \cdot 75$ & 147 & 308 \\
CB-9 & 5 & $1 \cdot 06$ & $7 \cdot 94$ & $1 \cdot 63$ & $7 \cdot 14$ & 217 & 452 \\
CB-10 & 5 & $0 \cdot 97$ & $9 \cdot 35$ & $1 \cdot 57$ & $7 \cdot 83$ & 235 & 406 \\
\hline
\end{tabular}

Abbreviations as in table 2 .

maximum frequencies for fine crackles were significantly higher than those for coarse crackles $(p<0.0001)$. Although there were significant differences in all the measured values between fine and coarse crackles, there was some overlap in the values for individual crackles between two groups.

Mean values for waveform and spectral measurements for each patient (table 3 and figs 4-6) show no overlap between fine and coarse crackles. Cut off values for each waveform measurement were $0.88,6 \cdot 77,1.37$, and $5.9 \mathrm{~ms}$ for a quarter cycle duration, $9 / 4$ cycle duration, initial deflection width, and two cycle duration respectively (figs 4 and 5). For fast Fourier analysis cut off values for peak and maximum frequencies were $288.5 \mathrm{~Hz}$ and $497 \mathrm{~Hz}$ respectively (fig 6).

The correlations between measures of pooled data are shown in table 4 . There was highly significant correlations between $1 / 4$ cycle duration and initial deflection width $(r=0.95)$ and between $9 / 4$ cycle duration and two cycle duration $(r=0.99)(p<0.0001)$. Measures of
Figure 4 Mean values of 1/4 cycle duration (left panel) and 9/4 cycle duration (right panel) of the crackles for each patient with idiopathic pulmonary fibrosis (IPF)-fine cracklesand with chronic bronchitis $(C B)$-coarse crackles.

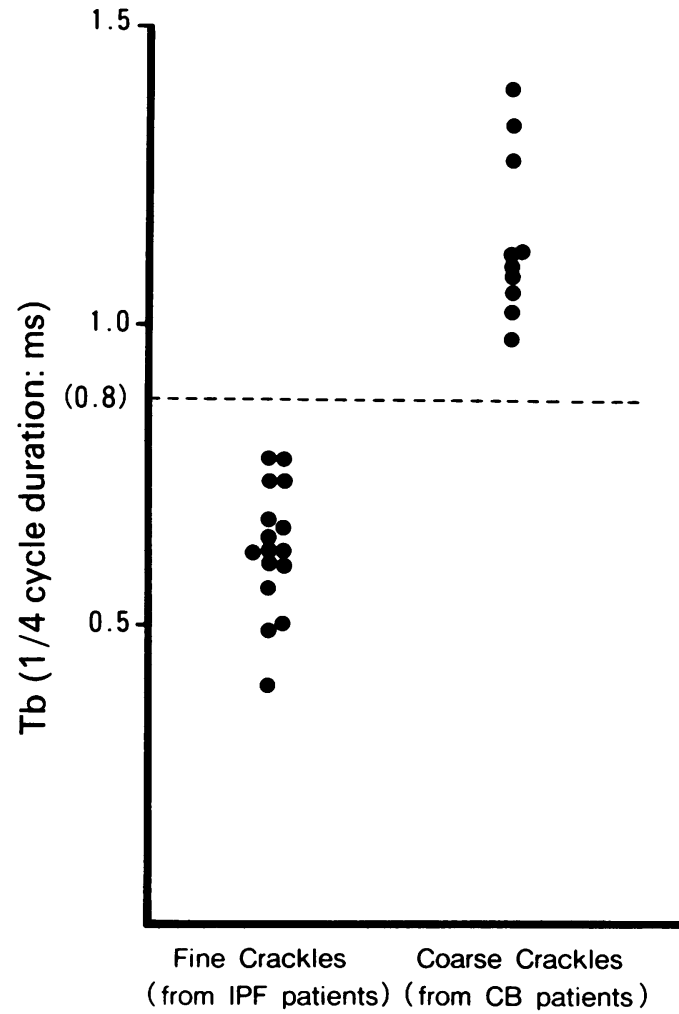

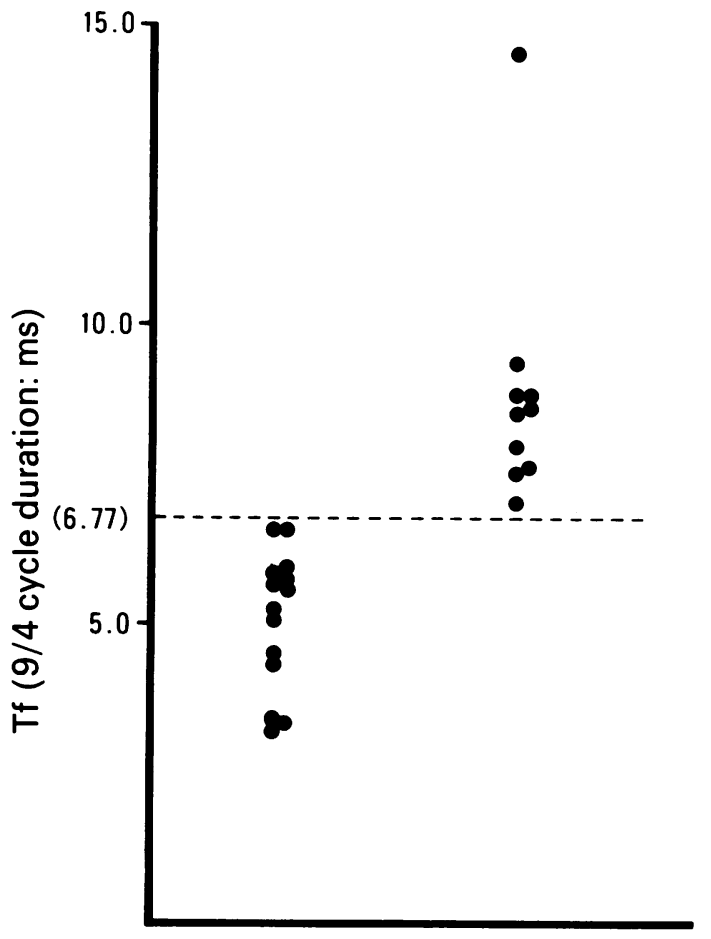

Fine Crackles Coarse Crackles (from IPF patients) (from CB patients) 
Figure 5 Mean values of initial deflection width (left panel) and two cycle duration (right panel) of the crackles for each patient with idiopathic pulmonary fibrosis (IPF)-fine cracklesand with chronic bronchitis $(C B)$-coarse crackles.

Figure 6 Mean values of peak frequency (left panel) and maximum frequency (right panel) of the crackles for each patient with idiopathic pulmonary fibrosis (IPF)-fine cracklesand with chronic bronchitis $(C B)$-coarse crackles.
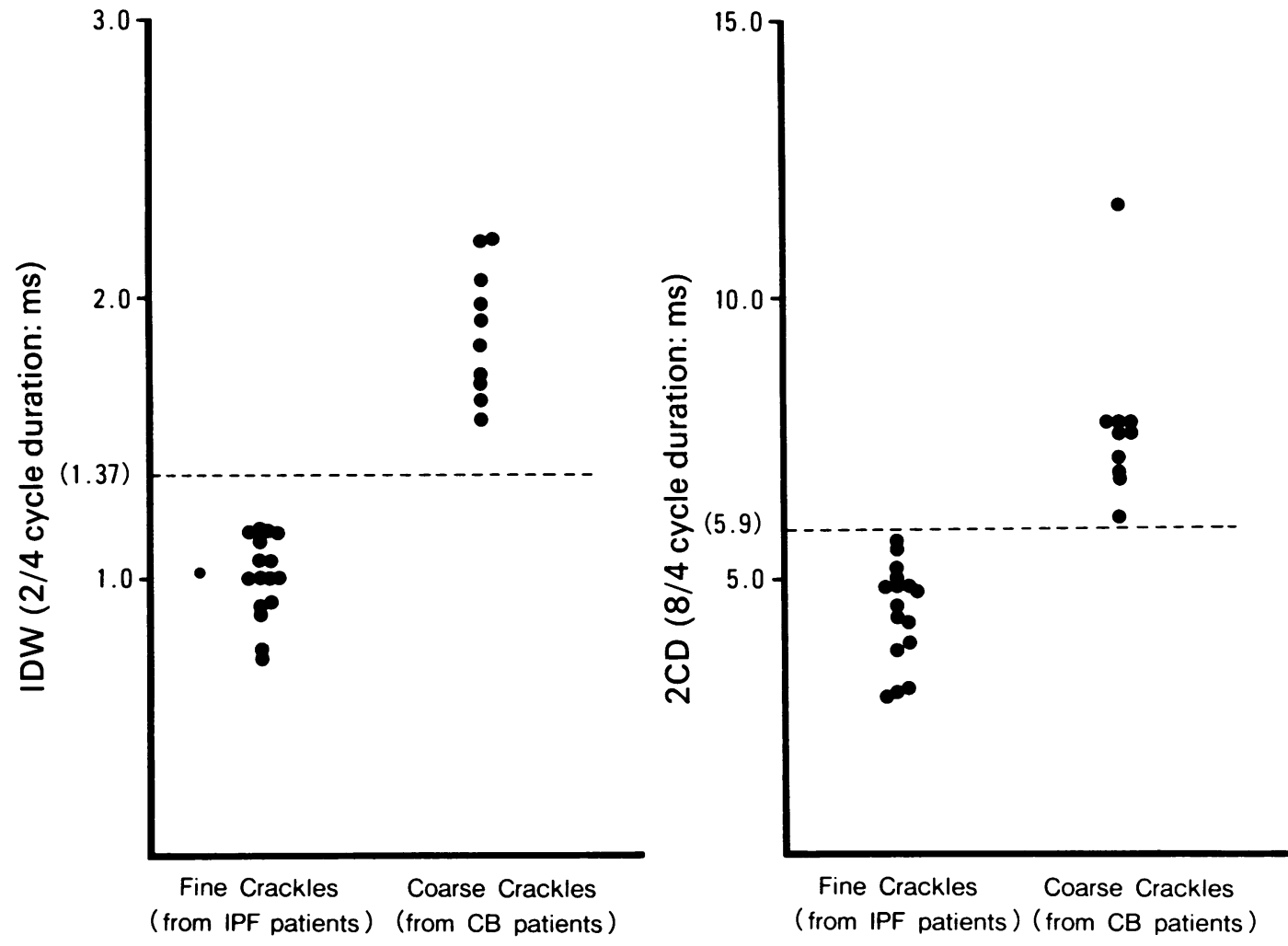

frequency (log peak frequency and log maximum frequency) also correlated significantly with waveform measures, as shown in the table (correlation coefficients between 0.66 and 0.87 ). When these correlation coefficients were compared, the log peak frequency and log maximum frequency correlated with $9 / 4$ cycle duration $(r=0.85,0.84)$ and two cycle duration $(r=0.87,0.86)$ better than with $1 / 4$ cycle duration $(r=0.66,0.77)$ and initial deflection width $(r=0.67,0.79 ; p<0.01)$.

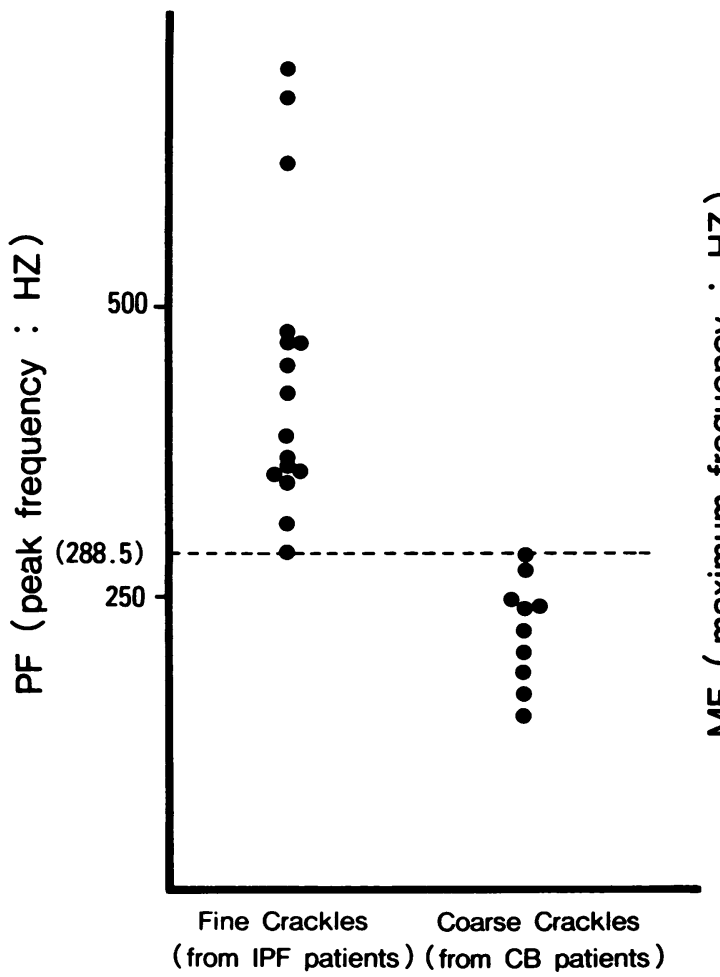

\section{Discussion}

Pulmonary auscultation is easily applied, noninvasive, and economical. The information obtained may reflect regional pathophysiological disturbances..$^{4-714}$ Despite these advantages its clinical usefulness has been questioned, especially with the development of modern diagnostic techniques. One reason is the lack of objectivity, which makes it difficult to assess the importance of auscultatory findings. Efforts to solve this problem have been

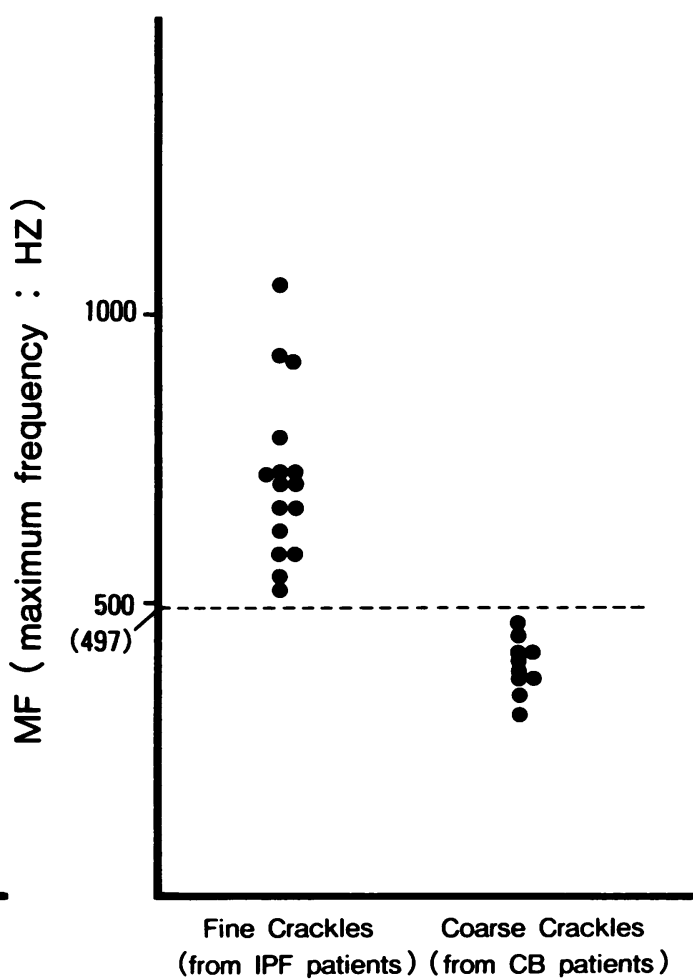


Table 4 Correlation coefficient ( $r$ ) matrix of parameters

\begin{tabular}{|c|c|c|c|c|c|c|}
\hline & $\log P F$ & $\log M F$ & $T b$ & $T f$ & $I D W$ & $2 C D$ \\
\hline $\begin{array}{l}\log (P F) \\
\log (M F) \\
\text { Tb } \\
\text { Tf } \\
\text { IDW } \\
2 \text { CD }\end{array}$ & 1.00 & $\begin{array}{l}0.87 \\
1.00\end{array}$ & $\begin{array}{c}-0.66^{\star} \\
-0.77^{\ddagger} \\
1.00^{\star}\end{array}$ & $\begin{array}{c}-0.85^{\star} \\
-0.84_{+}^{\dagger} \\
0.76 \\
1.00\end{array}$ & $\begin{array}{c}-0.67 \dagger \\
-0.79 \S \\
0.95 \\
0.77 \\
1.00\end{array}$ & $\begin{array}{c}-0.87 \dagger \\
-0.86 \S \\
0.79 \\
0.99 \\
0.79 \\
1.00\end{array}$ \\
\hline
\end{tabular}

All correlations were significant $(p<0.0001)$. Differences between pairs of correlation coefficients: ${ }^{\star} \mathrm{p}<0.005 ; \mathrm{tp}<0.005 ; \neq \mathrm{p}<0.025 ;$ §p $<0.05$.

Abbreviations as in table 2 . between the $1 / 4$ cycle duration and the initial deflection width $(r=0.95)$ and between the $9 / 4$ cycle duration and the two cycle duration $(r=0.99)$. These results indicate that the $1 / 4$ cycle and $9 / 4$ cycle duration ${ }^{11}$ and the initial deflection width and two cycle duration ${ }^{10}$ provide almost the same information. We recommend measuring the $1 / 4$ and $4 / 9$ cycle durations as this method is not affected by baseline drift, which makes the determination of initial deflection width and two cycle duration difficult. ${ }^{11}$ Holford reported a mean initial deflection width and two cycle duration of 0.92 and 6.02 respectively for fine crackles and of 1.25 and 9.32 for coarse crackles. ${ }^{10}$ Their initial deflection width value for fine crackles is similar to ours, whereas our value for coarse crackles is larger; the two cycle duration values for fine and coarse crackles were smaller in their study. They did not give precise information about how their patients were selected. Differences in selection of patients may account for the different results in the two studies. In some patients with idiopathic pulmonary fibrosis both inspiratory and expiratory crackles are heard. ${ }^{1819}$ Preliminary analysis of expiratory crackles from our patients with idiopathic pulmonary fibrosis showed that the crackles could be fine or coarse or due to a pleural friction rub. ${ }^{18}$ Inspiratory crackles heard in such patients ${ }^{18}$ might also contain coarse crackles or pleural friction rubs in addition to fine crackles. In our study, therefore the patients were selected carefully to minimise the likelihood that both types of crackle would occur in the same patient.

An additional interesting finding in this study was that the $9 / 4$ cycle duration and two cycle duration correlated much better with log peak frequency and $\log$ maximum frequency than did the $1 / 4$ cycle duration and initial deflection width (table 4 ). This shows that the $1 / 4$ cycle duration and initial deflection width yield information that is qualitatively different from that of the peak and maximum frequencies. Mori et $a l^{20}$ suggested that waveforms of crackles consist of two components, a starting segment and a decay segment. They speculated that the early starting segment usually contained the characteristics of a shock wave and that the decay segment might be determined by the characteristics of the pulmonary structure as a resonance apparatus, the whole crackle waveform being made from the combination of these two components. Our results are consistent with these ideas. The $1 / 4$ cycle duration and initial deflection width, measurements of the initial part of the crackle waveform, may represent information that comes from the site of origin of the sound rather than from the resonator.

In summary, we sampled fine crackles from selected patients with idiopathic pulmonary fibrosis and coarse crackles from patients with chronic bronchitis and obtained measurements of frequency and waveform analysis. Although the characteristics of individual crackles did not separate the two groups completely, the mean values for each patient separated the two groups clearly. These results suggest that spectral and waveform characteristics of crackles results suggest that waveform and fast Fourier analyses of several crackles from a patient will allow the two diseases to be separated.

There were highly significant correlations 
may help to improve the accuracy of pulmonary auscultation and to expand our knowledge of how crackles are generated.

This work was supported by a scientific research grant (62870032) from the Ministry of Education, Science, and Culture, Japan.

1 Nath AR, Capel LH. Inspiratory crackles-early and late. Thorax 1974;29:223-7.

2 Report of the ATS-ACCP ad hoc subcommittee on nomenclature. American Thoracic Society News 1977;3:5-6.

3 Loudon R, Murphy RL. Lung sounds. Am Rev Respir Dis 1984;130:663-73.

4 Forgacs $P$. The functional basis of pulmonary sounds. Chest 1978;73:399-405.

5 Forgacs P. Crackles and wheezes. Lancet 1967;ii:203-5.

6 Forgacs P. Gravitational stress in lung disease. $\mathrm{Br} \mathrm{J} \mathrm{Dis} \mathrm{Chest}$ 1974;68:2-10.

7 Nath AR, Capel LH. Inspiratory crackles and mechanical events of breathing. Thorax 1974;29:695-8.

8 Munakata $M$, Homma $Y$, Matsuzaki $M$, Ogasawara $H$ Tanimura K, Kusaka H, et al. Production mechanism of crackles in excised normal canine lungs. J Appl Physiol 1986;61:1120-5.

9 Murphy RLH Jr, Holford SK, Knowler WC. Visual lungsound characterization by time-expanded wave-form analysis. N Engl J Med 1977;296:968-71.
10 Holford SK. Discontinuous adventitious lung sounds: measurement, classification and modeling. $\mathrm{ScD}$ thesis, Massachusetts Institute of Technology, 1982.

11 Matsuzaki M, Homma Y. A new method for classifying discontinuous adventitious lung sounds. Jpn $J$ Thorac Dis 1983;21:134-40.

12 Hotelling $H$. The selection of variables for use in prediction with some comments on the general problem of nuisance parameters. Annals of Mathematical Statistics 1940; 11:271-83.

13 Murphy RL. Auscultation of the lung: past lessons, future possibilities. Thorax 1981;36:99-107.

14 Loudon R, Murphy RLH. Lung sounds. Am Rev Respir Dis 1984;130:663-73.

15 Homma M, Matsuzaki M, Ogasawara $H$, Munakata $M$. Phonopneumograph possible for real-time tracing. Computer and Biomedical Research 1985;18:502-9.

16 McKusick VA, Jenkins J, Webb GN. The acoustic basis of the chest examination. Am Rev Tuberc 1955;72:12-34.

17 Kraman SS. New tools in lung sound research. Semin Respir Med 1985:220-8.

18 Munakata $M$, Tanimura $K$, Ogasawara $H$, Matsuzaki $M$, Homma Y, Kawakami Y. Expiratory crackles in diffuse interstitial lung diseases. Ther Res 1985;3:660-6.

19 Walshaw MJ, Nisar M, Rearson MG, Calverley PMA, Earis JE. Expiratory lung crackles in patients with fibrosing alveolitis. Chest 1990;97:407-9.

20 Mori M, Kinoshita K, Morinari H, Shiraishi T, Koike S, Murao S. Waveform and spectral analysis of crackles. Thorax 1980;35:843-50. 